Vol 12, Issue 10, 2019

\title{
EFFECT OF NOISE STRESS-INDUCED NEUROBEHAVIORAL CHANGES ON WISTAR ALBINO RATS
}

\section{ARCHANA ARJUNAN, RAVINDRAN RAJAN*}

Department of Physiology, Dr. ALM PG IBMS, University of Madras, Chennai, Tamil Nadu, India. Email: ravindran89@gmail.com

Received: 09 July 2019, Revised and Accepted: 30 July 2019

\author{
ABSTRACT \\ Objective: The study aims to investigate the effect of acute noise stress on cognitive functions in male Wistar albino rats
}

Methods: Adult albino rats were randomly divided into two groups. Each group contains six animals. Rats exposed to acute noise stress (100 dB/4 h) were compared with control animal and assessed for cognition using T-maze, hole-board test, open-field test, marble burying test, and social interaction behavior.

Results: The rats exposed to acute noise stress showed the significance $(\mathrm{p}<0.05)$ of behavioral alterations such as impaired learning and memory, memory retention, increased fear and anxiety, obsessive-compulsive behavior, social avoidance, and decreased social interaction.

Conclusion: The results report that acute noise stress affects the cognition, and it became chronic may confer the increased risk of neurodegenerative disorders.

Keywords: Cognition, Learning, Noise, Stress, Neurodegenerative disease, Social interaction.

(C) 2019 The Authors. Published by Innovare Academic Sciences Pvt Ltd. This is an open access article under the CC BY license (http://creativecommons. org/licenses/by/4. 0/) DOI: http://dx.doi.org/10.22159/ajpcr.2019.v12i10.34843

\section{INTRODUCTION}

Stress is an unavoidable circumstance in our life. Noise is gradually becoming the most pervasive environmental and occupational stress pollutant in a modern era [1]. In our modern lifestyle, using of various automobiles, urban traffic, household appliances, media [2] industrialization, Using weapons in Military and Army forces which increase the noise stress/pollution to the individuals who work in their environment [3]. Noise has been defined as unwanted sound. It consists of unpleasant obtrusive annoying, distracting, or persistent sounds that interfere with sleep or the ability to concentrate or enjoy life [4]. The World Health Organization stated that "Noise must be recognized as a major threat to human wellbeing" [5]. If the noise level exceeded $90 \mathrm{dBA}$, it becomes a source of stressor [6] and affects the health by both physical and psychological. However, the damaging effects of noise are not limited to the auditory system but rather extend too many other systems. Acute and chronic loud noise exposure generates excessive free radicals and causes disorders involving extra-auditory organs such as nervous, endocrine, and cardiovascular systems [7]. Specifically, neural tissue is very sensitive to oxidative stress due to the brain cells are more vulnerable to free radical damage caused by lipid peroxidation compared to other tissues, owing to their high level of unsaturated fatty acids [8]. Maladaptive effects of noise stress enhance the ROSinduced behavioral changes and neurodegenerative disease [1]. From this, it is planned to study the effect of acute noise on cognition in a noise-induced animal model.

\section{METHODS}

\section{Animals}

Experimental animals were all healthy and weighed about 140-180 g. The animals were reared in the Animal House of the Institute, University of Madras, Taramani, Chennai, India, and all the animals were maintained under standard laboratory conditions housed 3 per cage (29 $\mathrm{cm} \times 22 \mathrm{~cm} \times 14 \mathrm{~cm}$ ) and constant ambient temperature with 12 -h dark photoperiod; the rats were allowed free access to food and water. Appropriate ethical clearance was obtained for this work from the Institutional Animal Ethical Committee (IAEC no. 02/06/2019 dated
$12 / 03 / 2019)$ prior to the experiments. Animals were divided into two groups, with six animals in each group.

- Group I - Control

- Group II - Acute noise stress (100 dBA - 4 h/1 day).

\section{Noise stress induction}

When noise exposure exceeds $100 \mathrm{dBA}$, noise becomes a stressor. Noise was produced by two loudspeakers (15 W), driven by a white noise generator $(0-26 \mathrm{kHz})$, and installed $30 \mathrm{~cm}$ above the cage. The noise level was set at $100 \mathrm{~dB}$ uniformly throughout the cage and monitored by a sound-level meter D2023 (S.NO-F02199: Cygnet Systems, Gurgaon, Haryana, India). Animals were then exposed for $4 \mathrm{~h} / 1$ day. To avoid the influence of handling stress on evaluation, the effects of noise exposure in control animals were kept in the above-described cage during the corresponding period of time, without noise stimulation [8].

\section{Behavioral assessment}

\section{T-maze}

It is shaped like the letter T, providing the rodent, with a straightforward choice. T-mazes are used to study how the rodents function with memory and spatial learning through applying various stimuli. The T-maze consists of a start box $(16.5 \mathrm{~cm} \times 16.5 \mathrm{~cm})$, stem (arm 1) length is $71.1 \mathrm{~cm}$, goal area (arm 2 and 3) lengths are $45.7 \mathrm{~cm}$, and arm width is $10.2 \mathrm{~cm}$. The stem and start box are separated by a sliding door, and T-maze is kept in a dimly lit, sound-attenuated room. Behavioral evaluation was performed in three phases. (i) Orientation session: This session was to familiarize the rats with the T-maze. Rats are semi-starved for $24 \mathrm{~h}$ and allowed to explore the T-maze for $15 \mathrm{~min}$. Rats were placed in the start box for $30 \mathrm{~s}$. Sliding door was opened to allow rats to explore the T-maze for $15 \mathrm{~min}$ and to eat food pellets in each goal area. Rats were then returned to the start box. (ii) Learning performance test: After the $1^{\text {st }}$ day of orientation, the rats were trained for the rewarded alternation task. Learning performance test followed the same procedure as the orientation session except that one arm is baited. The rat has to alternate the arms for the reward. The placement of the reward in the subsequent trial will be opposite to the rats' previous arm entry. Each rat received ten trials per day. The intertrial interval was $30 \mathrm{~s}$, during which the maze was cleaned with $70 \%$ alcohol 
to remove odor cues. The number of days each rat took for $80 \%$ correct choice (eight correct entries to the baited arm out of ten trials), and the time taken to reach the goal area was noted. (iii) Retention test: 2 days after the last training session, memory retention test was carried out. Rats were given ten trials continuously, with an intertrial interval for $30 \mathrm{~s}$. Numbers of errors, i.e., entry into the non-rewarded arm is recorded in ten trials, were noted [9].

\section{Hole-board test}

The hole-board apparatus consisted of a wooden, gray box, measuring $68 \mathrm{~cm} \times 68 \mathrm{~cm}$. The walls were $40 \mathrm{~cm}$ high, and the box was raised $28 \mathrm{~cm}$ above the ground on a metal stand. Four holes $(4 \mathrm{~cm}$ in diameter) were cut into the floor of the apparatus; each hole was 28 $\mathrm{cm}$ from a corner of the box along the diagonal from the corner to the center. The stand of the apparatus was open on all sides, allowing the floor or objects to be dimly lit. At the beginning of each trial, a subject was placed in one corner of the apparatus (always the corner closest to the door of the room), facing the center of the arena. Each trial lasted $10 \mathrm{~min}$. At the end of the trial, the subject was immediately placed into a carrying box and returned to the home cage. Between each trial, the floor and walls of the apparatus were cleaned with $70 \%$ alcohol solution. During each 10-min trial, head-dip: the animal places its head into one of the holes, to a minimum depth such that the ears were level with the floor of the apparatus (a new bout of head-dipping was recorded if the animal raised its head fully out of the hole before resuming); grooming, rearing, and fecal pellets' behavior patterns were recorded [10].

\section{Marble burying test}

Marble burying test done by polycarbonate rat cages (26 $\mathrm{cm} \times 48 \mathrm{~cm} \times 20 \mathrm{~cm}$ ) with fitted filter top covers, fresh, unscented mouse bedding material to each cage to a depth of $5 \mathrm{~cm}$ and level bedding surface by inserting another cage of the same size onto the surface of the bedding, and placed standard glass toy marbles (assorted styles and colors, $15 \mathrm{~mm}$ diameter, $5.2 \mathrm{~g}$ in weight) gently on the surface of the bedding in 5 rows of 4 marbles. Rats were placed into a corner of the cage containing marbles, being careful to place the mouse on bedding as far from marbles as possible, and place the filter top cover on the cage. Withhold food and water during the test. Allow rats to remain in the cage undisturbed for $30 \mathrm{~min}$. Score a marble as buried if two-thirds of its surface area is covered by bedding. Average scores for the number of marbles buried for each mouse [11]

\section{Social interaction task}

The social preference test was conducted in a rectangular, threechambered box (a center compartment of $20 \mathrm{~cm} \times 35 \mathrm{~cm} \times 35 \mathrm{~cm}$ with a left and aright compartment of $30 \mathrm{~cm} \times 35 \mathrm{~cm} \times 35 \mathrm{~cm}$ ) fabricated from opaque gray polycarbonate. The dividing walls had retractable doorways allowing access to each chamber. The test rat was placed in the middle chamber and allowed to explore the entire apparatus for $5 \mathrm{~min}$. Each of the two side chambers contained an empty wire cage. The wire cages were $10 \mathrm{~cm}$ in height, with a bottom diameter of $9 \mathrm{~cm}$ and bars spaced $1 \mathrm{~mm}$ apart. A weighted plastic cone was placed on the top of each cage to prevent climbing by the test rats. Four sets of wire cages were used during the experiment, and all of the cages were washed with water and dried properly between each use. For habituation to the wire cage, each novel pre-pubertal male rat used in the social interaction test had been previously placed in the wire cage in the apparatus without the test rat for 5 min on 3 consecutive days preceding the social test. On the day after the last habituation session, a test rat was placed in the center compartment and allowed to explore the entire apparatus for $10 \mathrm{~min}$. An unfamiliar rat was placed in one of the wire cages located on either side of the social test box during the 10 min session. A rectangular colored object was placed in the other wire cage on the other side of the box. The location of the stranger and the object in the left and right sides of the chamber was counterbalanced for different animals. Placing the strange rat in a wire cage prevented direct physical contact between the rats and ensured that the social approach was only initiated by the test rat. The time spent sniffing each wire cage was videorecorded and manually scored to evaluate the level of preference for the unfamiliar rat as compared to the object. The entire apparatus was cleaned with water and dried thoroughly between each tested rat [12].

\section{Open-field test}

Open-field behavior is a simple test to evaluate the locomotion and emotional status of the animals. The apparatus used was a large rectangular box $(100 \mathrm{~cm} \times 100 \mathrm{~cm})$ made up of $40 \mathrm{~cm}$ high plywood walls. The floor consisted of a clean dark plastic material with a grid painted in white dividing the field into $25(5 \times 5)$ equal squares. Illumination was provided by a $60 \mathrm{~W}$ bulb placed $100 \mathrm{~cm}$ above the center of the field. A weak cider vinegar solution (10\%) was used to clean the apparatus before the introduction of each animal. The rat was placed in one corner of the apparatus, and its behavior was observed for $5 \mathrm{~min}$ [13].

\section{Statistical analysis}

Data were presented, in the form of the bar diagram with mean \pm standard deviation. All data are analyzed with the SPSS for Windows statistical package (version 20.0, SPSS Institute Inc., Cary, North Carolina, USA). Statistical significance between two groups was determined by paired Student's t-test, and $\mathrm{p}<0.05$ was considered as statistically significant.

\section{RESULTS AND DISCUSSION}

The scientific understanding of "stress" and its ramifications for the organism has continually evolved [14].Stress is a global menace fortified by the advancement of industrialization and elicited by a variety of factors such as environmental, social, or pathological phenomenon from life [15]. Stressors have a major influence upon mood, our sense of well-being, behavior, and health. The actual or perceived threat to an organism is referred to as the "stressor," and the response to the stressor is called the "stress response." Although stress responses evolved as adaptive processes, Selye observed that severe, prolonged stress responses might lead to tissue damage and disease [16]. Especially, stress initiates a series of neuronal responses and can lead to the development of behavioral disorders associated with cognitive impairments, depression, and anxiety [16].

In this study, $\mathrm{T}$-maze showed that increased latency, number of days taken to the correctchoiceand reduced correct choice including, reduced retention memory in acute noise stress animals when compared to the control animals (Fig. 1). Acute stress can affect synaptic plasticity and cognition by acting in different brain regions. The neuroendocrine stress cascade, comprising the hypothalamic-pituitary-adrenocortical axis, begins with the release of adrenocorticotropic hormone in the medial parvocellular paraventricular nucleus, which travels to the anterior pituitary, where they can access corticotropes [14]. An increased concentration of corticosterone in stressed rats accompanied by higher blood glucose level indicates that stress-induced excess productions of glucocorticoids affected glucose metabolism [17]. The possible mechanism is glucocorticoids acts via glucocorticoid receptors and mineralocorticoid receptor and increases the extracellular glutamate levels and affect the excitatory transmission. Glucocorticoids were found as a common mediating mechanism for both the facilitating and impairing actions of stress in different memory processes and phases. Among the brain regions implicated, the hippocampus, amygdala, and prefrontal cortex were highlighted as critical for the mediation of stress effects [18]. In the hippocampus, stress was systematically shown to impair long-term potentiation while facilitating long-term depression [19].

In the hole-board task, the acute noise group showed the increased head dipping, grooming, rearing, and fecal pellets when compared to the control animals (Fig. 2). In marble burying task, acute noise stress 


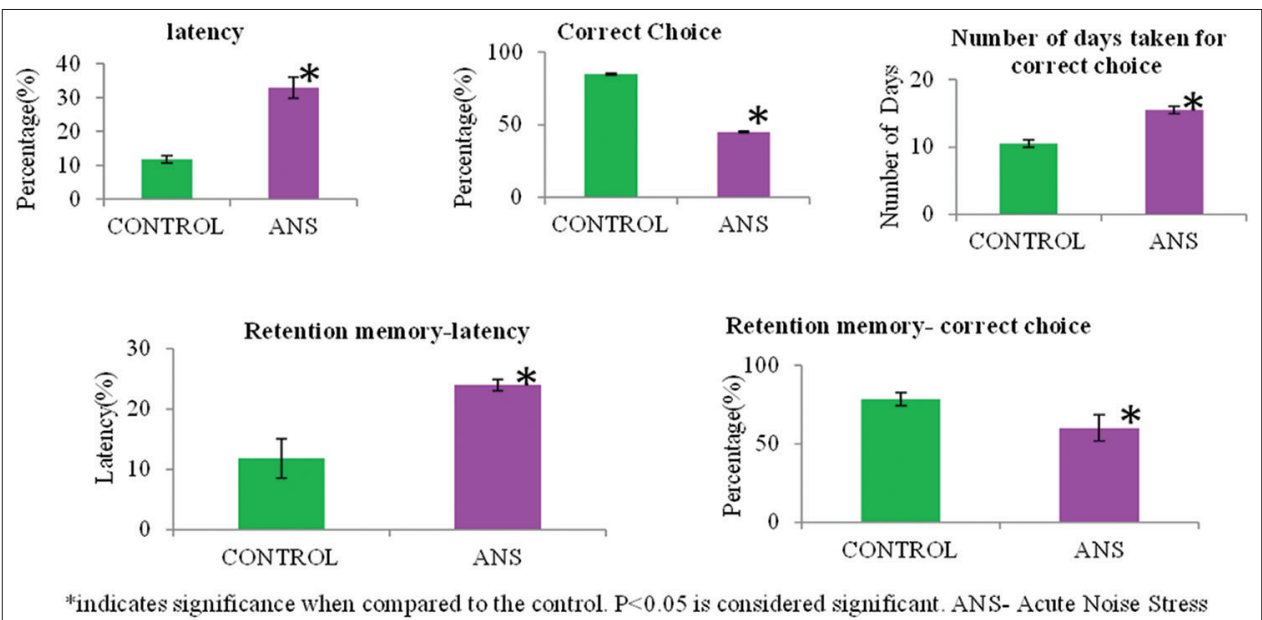

Fig. 1: T-maze

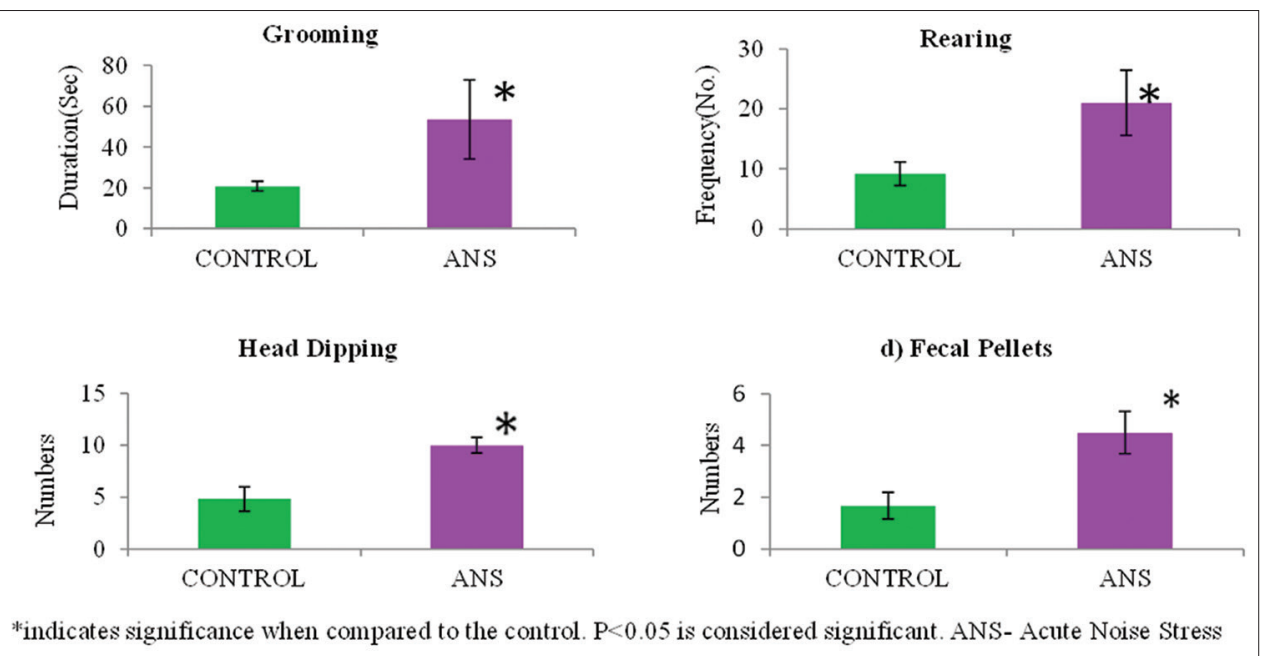

Fig. 2: Hole-board test

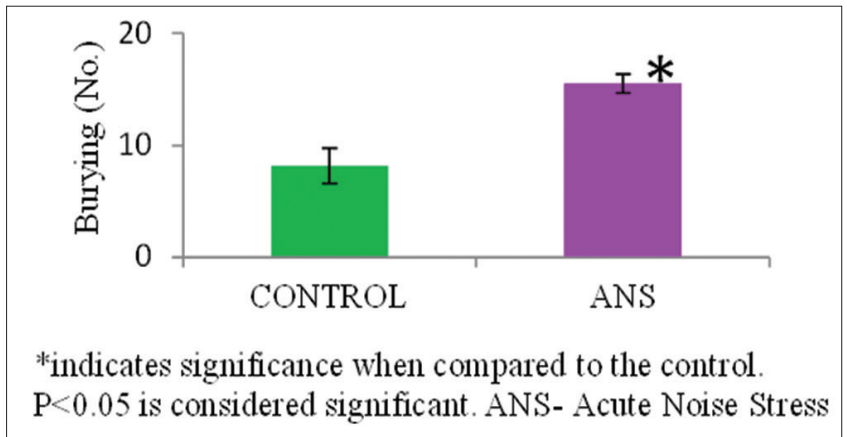

Fig. 3: Marble burying test

animals showed a significantly increased marble burying activity when compared to control animals (Fig. 3). In social interaction task with familiar animal, acute noise stress-induced animals showed a significantly increased time spent in a familiar compartment and decreased time spent with an unfamiliar animal when compared to control animals (Fig. 4). The bed nucleus of the stria terminalis and the amygdala receive projections from the basolateral amygdala (BLA) and project, in turn, to downstream target areas that mediate many of the behavioral, autonomic, and electrophysiological consequences of fear and anxiety. High level of glucocorticoids increases the excitation of amygdala neurons by decreasing gamma-aminobutyric acid (GABA) level and also increasing the cytosolic calcium which triggers cytoarchitecture changes in BLA neurons [20]. And also, increased proinflammatory cytokine TNF- $\alpha$ and corticosterone may contribute to depression [21]. Earlier studies reported that acute stress, an increase in anxiety-like behavior, by triggers an increase in spine-density in principal neurons of the BLA [22]. Social avoidance is a common symptom in various anxiety disorders, including social anxiety, panic, and generalized anxiety. Haller reported that single stressors induce long-lasting social avoidance in rats [23].

In the open-field test, acute noise-exposed animals showed an increased time spent in peripheral squares when compared to the control animals which decreased the locomotor activity in acute stress animals (Fig. 5). Brain regions participate in motor control, such as motor cortex, cerebellum, and basal ganglia. The dopaminergic system is a major neurotransmitter involved in movement and locomotion via the basal ganglia. The cerebellum is known to contribute to motor control and coordination activity by integrating motor output with ongoing sensory feedback. During noise, increased dopamine facilitated that free radical damage in the cerebellum leads to impaired motor coordination. And also, cerebellar Purkinje cells release inhibitory neurotransmitter GABA which reduces the transmission of impulses. Activation of glutamatergic and GABAergic neurotransmission systems which also alter the motor coordination [1]. 


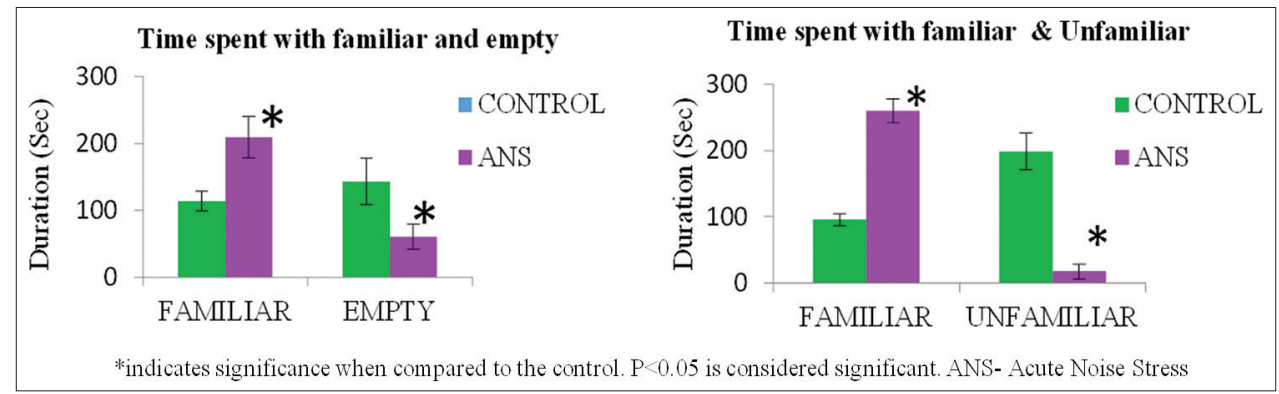

Fig. 4: Social interaction task

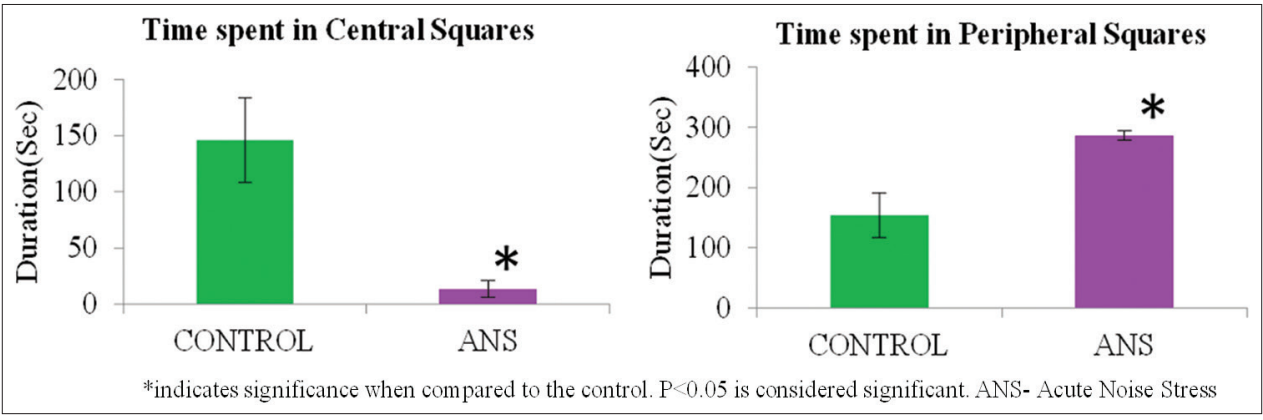

Fig. 5: Open-field test

\section{CONCLUSION}

Environmental challenges are part of our daily lives. In many instances, challenges can trigger stress responses in an individual. Noise becomes a hazard to the physical, mental, and social well-being of an individual who works in a noisy environment or who is vulnerable to exposure to noise. The present study revealed that acute noise stress altered the locomotor, fear and anxiety, learning and memory, social interaction, and obsessive and compulsive behavior of the rats. This could be linked to increased corticosterone and may be oxidative stress, which leads to alter neurotransmitter level, molecular gene expression, and cytokine level in the brain.

\section{ACKNOWLEDGMENT}

The authors are grateful to the Department of Physiology, University of Madras, for providing the infrastructure to conduct the research. The authors would like to acknowledge Dhiraj Kumar Sah, Research Scholar, Department of Biomedical Sciences, Chonnam National University, South Korea, for helping out English and the final correction of the manuscript.

\section{AUTHOR CONTRIBUTION}

The corresponding author has designed the work and critical revision of the manuscript. The first author carried out behavioral assessment and paper writing.

\section{CONFLICTS OF INTEREST}

The authors declare that they no conflicts of interest concerning this research article.

\section{REFERENCES}

1. Wankhar W, Srinivasan S, Sundareswaran L, Wankhar D, Rajan R, Sheeladevi R, et al. Role of Scoparia dulcis Linn on noise-induced nitric oxide synthase (NOS) expression and neurotransmitter assessment on motor function in wistar albino rats. Biomed Pharmacother 2017;86:475-81

2. Gai Z, Li K, Sun H, She X, Cui B, Wang R, et al. Effects of chronic noise on mRNA and protein expression of CRF family molecules and its relationship with p-tau in the rat prefrontal cortex. J Neurol Sci
2016:368:307-13.

3. Henderson D, Bielefeld EC, Harris KC, Hu BH. The role of oxidative stress in noise-induced hearing loss. Ear Hear 2006;27:1-9.

4. Jamir L, Nongkynrih B, Gupta SK. Community noise pollution in urban India: Need for public health action. Indian J Community Med $2014 ; 39: 8-12$

5. Fink DJ. What is a safe noise level for the public? Am J Public Health 2017;107:44-5.

6. Srinivasan S, Wankhar W, Rathinasamy S, Rajan R. Neuroprotective effects of Indigofera tinctoria on noise stress affected wistar albino rat brain. J Appl Pharm Sci 2015;5:58-65.

7. Manikandan S, Devi RS. Antioxidant property of alpha-asarone against noise-stress-induced changes in different regions of rat brain. Pharmacol Res 2005;52:467-74.

8. Samson J, Sheela Devi R, Ravindran R, Senthilvelan M. Effect of noise stress on free radical scavenging enzymes in brain. Environ Toxicol Pharmacol 2005;20:142-8.

9. Deacon RM, Rawlins JN. T-maze alternation in the rodent. Nat Protoc 2006;1:7-12.

10. Brown GR, Nemes C. The exploratory behaviour of rats in the holeboard apparatus: Is head-dipping a valid measure of neophilia? Behav Processes 2008:78:442-8.

11. Angoa-Pérez M, Kane MJ, Briggs DI, Francescutti DM, Kuhn DM. Marble burying and nestlet shredding as tests of repetitive, compulsivelike behaviors in mice. J Vis Exp 2013;82:50978.

12. Castro JE, Diessler S, Varea E, Márquez C, Larsen MH, Cordero MI, et al. Personality traits in rats predict vulnerability and resilience to developing stress-induced depression-like behaviors, HPA axis hyper-reactivity and brain changes in pERK1/2 activity. Psychoneuroendocrinology 2012;37:1209-23

13. Saillenfait AM, Vannier B. Methodological proposal in behavioural teratogenicity testing: Assessment of propoxyphene, chlorpromazine, and Vitamin A as positive controls. Teratology 1988;37:185-99.

14. Myers B, McKlveen JM, Herman JP. Glucocorticoid actions on synapses, circuits, and behavior: Implications for the energetics of stress. Front Neuroendocrinol 2014:35:180-96.

15. Malathi S, Vidyashree HM, Rajan R. Role of Michelia champaca in memory enhancement and acute noise stressed male wistar albino rats. Int J Pharm Pharm Sci 2018;10:129-35.

16. Schneiderman N, Ironson G, Siegel SD. Stress and health: Psychological, behavioral, and biological determinants. Annu Rev Clin Psychol 2005;1:607-28.

17. Sarjan HN, Yajurvedi HN. Efficacy of an active compound of the herb, ashwagandha in prevention of stress induced hyperglycemia. Int $\mathrm{J}$ 
Pharm Pharm Sci 2018;10:44-9.

18. Sandi C, Pinelo-Nava MT. Stress and memory: Behavioral effects and neurobiological mechanisms. Neural Plast 2007;2007:78970.

19. Sandi C. Glucocorticoids act on glutamatergic pathways to affect memory processes. Trends Neurosci 2011;34:165-76.

20. Mitra R, Sapolsky RM. Acute corticosterone treatment is sufficient to induce anxiety and amygdaloid dendritic hypertrophy. Proc Natl Acad Sci U S A 2008;105:5573-8.

21. Anacker C, Cattaneo A, Luoni A, Musaelyan K, Zunszain PA,
Milanesi E, et al. Glucocorticoid-related molecular signaling pathways regulating hippocampal neurogenesis. Neuropsychopharmacology 2013;38:872-83

22. Kedia S, Chattarji S. Marble burying as a test of the delayed anxiogenic effects of acute immobilisation stress in mice. J Neurosci Methods 2014;233:150-4

23. Haller J, Bakos N. Stress-induced social avoidance: A new model of stress-induced anxiety? Physiol Behav 2002;77:327-32. 